\title{
Using the CA Opioid Overdose Surveillance Dashboard to track opioid overdose deaths
}

\author{
Jaynia A. Anderson, Natalie Demeter, Mar-y-sol Pasquiers, Stephen Wirtz
}

Safe and Active Communities Branch, California Department of Public Health, Sacramento, California, United States

Objective

Demonstrate the use of timely, actionable data from a data visualization tool, the California Opioid Overdose Surveillance Dashboard, which integrates statewide, geographic- and demographic-specific data, by describing the changes in opioid overdose deaths in California.

\section{Introduction}

California continues to face a serious public health crisis with the opioid epidemic having substantial health and economic impacts. The epidemic is dynamic and rapidly changing, involving both prescription opioids influenced by prescribing and dispensing patterns as well as illicit opioids influenced by the availability of heroin and recently, the increased availability of fentanyl. The complexity of the issue necessitates data-informed actions through multi-sector, strategic collaboration at both the state and local levels to address the problem comprehensively. With nearly 2,000 opioid overdose deaths per year and wide variation of overdose rates across counties and demographic groups, there is a need for integrated, timely, actionable data for use by state policy makers, local opioid safety coalitions, media, community stakeholders, and the public to monitor and combat this dynamic epidemic at the state and local level. Using fatality data from the California Opioid Overdose Surveillance Dashboard [1], the opioid overdose epidemic is described along with the differential geographic and demographic impacts.

\section{Methods}

As part of California Department of Public Health's Prevention for States grant funded by the Centers for Disease Control and Prevention, the California Opioid Overdose Surveillance Dashboard was developed as a data tool to provide enhanced visualization and integration of non-fatal and fatal opioid-involved overdose data and opioid prescription data. The dashboard was built on an open source RStudio server using Shiny, an R package that provides a framework for building web applications. Data incorporated on the dashboard include emergency department visits, hospitalizations, fatalities, and prescriptions related to opioid overd oses among California residents, presented in raw counts, crude rates, and age-adjusted rates at the state, county, and zip code levels, as well as by sex, age, and race/ethnicity. Overdose deaths are identified using ICD-10 (International Classification of Diseases, $10^{\text {th }}$ Revision) codes X40-X44, X60-X64, X85, Y10-Y14, and T40.0-T40.6, recorded in the underlying cause of death and multiple cause of death fields on death certificates. Fentanyl overdose deaths are identified using a text search on contributing cause of death fields on death certificates. Using data from the California Opioid Overdose Surveillance Dashboard, we present one perspective of the epidemic by using 2017 death data to describe the changing trend and geographic and demographic variation of prescription drug, heroin, and fentanyl overdose deaths.

\section{Results}

Overall trends from 2011-2017 show that deaths due to opioid overdoses have increased. Prescription drug overdose death rates have slightly decreased by $6 \%$ from $3.93 / 100,000$ in 2011 to $3.7 / 100,000$ in 2017 . Heroin overdose death rates have increased by $89 \%$ from $0.90 / 100,000$ in 2011 to $1.70 / 100,000$ in 2017.

Fentanyl overdose death rates have increased by $320 \%$ from $0.25 / 100,000$ in 2011 to $1.05 / 100,000$ in 2017 . The highest rates of prescription opioid overdose deaths are primarily concentrated in northern rural counties, while the highest rates of heroin and fentanyl overdose deaths are more dispersed throughout the state with many coastal counties showing higher rates of overdose deaths (Figure 1). Prescription opioid overdose deaths are concentrated among older ages showing highest rates among 55 to 59 year olds $(8.27 / 100,000)$. In contrast, heroin and fentanyl overdose death rates are concentrated among younger ages with the highest rates seen among 25 to 29 year olds, 4.54/100,000 and 2.78/100,000, respectively (Figure 2). Males died from prescription opioid, heroin, and fentanyl overdoses at significantly higher rates than females. Prescription opioid and fentanyl overdose death

SDS Annual Conference Proceedings 2019. This is an Open Access article distributed under the terms of the Creative Commons AttributionNoncommercial 4.0 Unported License (http://creativecommons.org/licenses/by-nc/3.0/), permitting all non-commercial use, distribution, and reproduction in any medium, provided the original work is properly cited. 
rates (11.5/100,000 and 4.80/100,000, respectively) are significantly higher among Native Americans compared to other races/ethnicities (Table 1). Non-Hispanic whites had significantly higher prescription opioid and heroin overdose death rates (6.90/100,000 and 2.96/100,000, respectively) compared to non-Hispanic black, Hispanic, and Asian residents of California.

\section{Conclusions}

Fatality data from 2017 show the characteristics of the opioid overdose epidemic in California are changing. While still high, overdose deaths from prescription opioids, seen primarily in older age groups and northern rural California, are slightly declining. Concurrently, we are seeing sharp rises in heroin and fentanyl overdose death rates among younger adults throughout the state. Regardless of any change in trend, there remain clear disparities in overdose death rates by race/ethnicity; with Native Americans having the highest rates for both prescription and illicit opioids, and non-Hispanic whites have higher rates of prescription opioid and heroin overdose deaths.

Given the varying demographic and geographic impacts based on the type of opioid, as demonstrated with the use of death data, there needs to be targeted data- informed interventions to address and prevent prescription and illicit opioid overdoses. Death data is just one perspective on the epidemic, other data sources (emergency department visits, hospitalizations, and prescriptions) are needed complete the picture to truly provide a robust data-informed approach. The California Opioid Overdose Surveillance dashboard integrates these multiple data sources and serves as a valuable tool in providing specific and timely data to inform approaches and interventions at the state and local level in continuing to fight California's opioid overdose epidemic. The enhanced visualization, geographic- and demographic-specific data, and increasingly timely data allow for state and local policy makers, local opioid safety coalitions, and community stakeholders to track the dynamics and impact of the epidemic and to identify those who are most vulnerable and differentially impacted.

\section{Acknowledgement}

The California Opioid Overdose Surveillance Dashboard is funded by the Centers for Disease Control and Prevention's Prevention for States grant.

\section{References}

1. California Opioid Overdose Surveillance Dashboard https://discovery.dev.cdph.ca.gov/CDIC/ODdash/

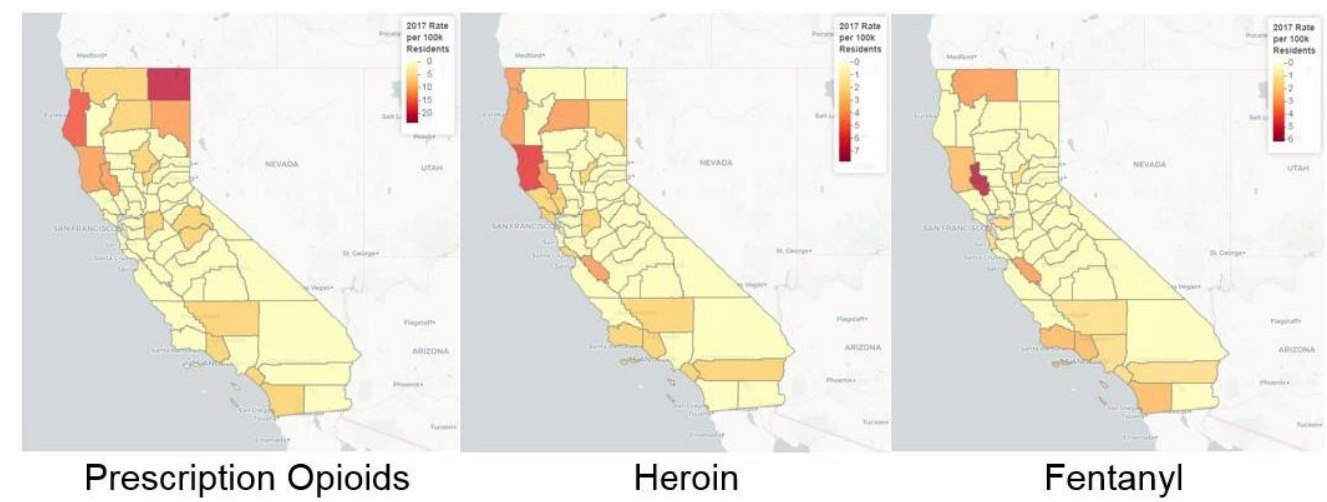

Figure 1. Overdose deaths from prescription opioids, heroin, and fentanyl in California, 2017 
ISDS 2019 Conference Abstracts

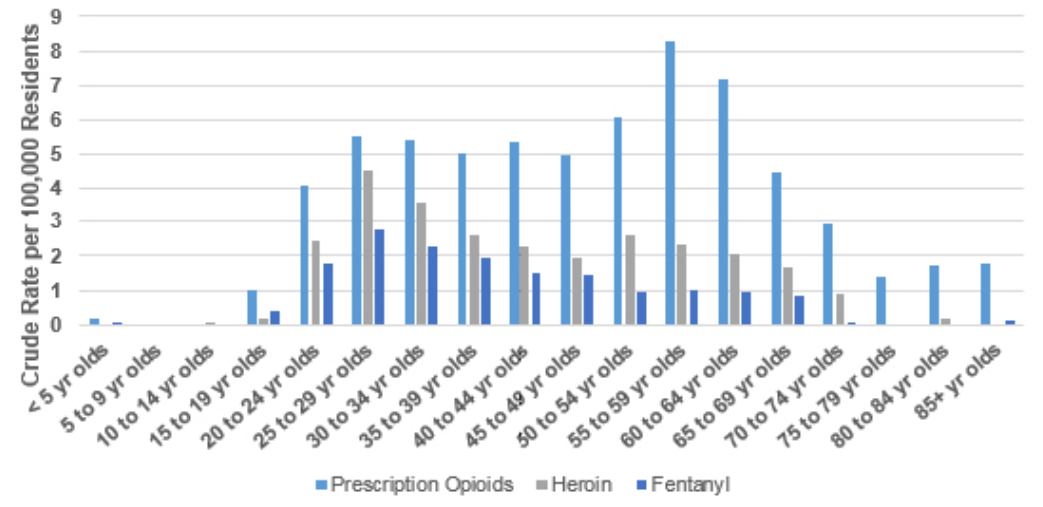

Figure 2. Overdose Death Rates from Prescription Opioids, Heroin, and Fentanyl by 5-Year Age Groups in California, 2017

Table 1. Overdose death rates from prescription opioids, heroin, and fentanyl by sex and race/ethnicity per 100,000 residents, California, 2017

\begin{tabular}{|c|c|c|c|}
\hline & Prescription Opioids & Heroin & Fentanyl \\
\hline & Crude Rate $(95 \% \mathrm{CI})$ & Crude Rate $(95 \%$ CI) & Crude Rate (95\% CI) \\
\hline \multicolumn{4}{|l|}{ Sex } \\
\hline Male & $5.22(4.90,5.55)$ & $2.96(2.72,3.21)$ & $1.75(1.57,1.95)$ \\
\hline Female & $2.67(2.45,2.91)$ & $0.65(0.55,0.78)$ & $0.42(0.34,0.52)$ \\
\hline \multicolumn{4}{|l|}{ Race/Ethnicity } \\
\hline White & $6.90(6.59,7.33)$ & $2.96(2.69,3.25)$ & $1.67(1.47,1.89)$ \\
\hline Black & $4.46(3.67,5.38)$ & $1.93(1.42,2.56)$ & $1.43(1.00,1.99)$ \\
\hline Hispanic & $2.10(1.89,2.35)$ & $1.17(1.01,1.35)$ & $0.75(0.62,0.90)$ \\
\hline Native American & $11.52(7.46,17.07)$ & $5.76(3.00,10.04)$ & $4.80(2.34,8.79)$ \\
\hline Asian & $0.74(0.55,0.99)$ & $0.32(0.20,0.50)$ & $0.23(0.13,0.38)$ \\
\hline
\end{tabular}

